\title{
VALIDEZ FACTORIAL DE LA ESTRUCTURA DEL CUESTIONARIO BREVE DE BURNOUT (CBB) EN UNA MUESTRA DE DOCENTES EN ARAGÓN
}

\author{
JESÚS MONTERO $^{1}$, JAVIER GARCÍA-CAMPAYO ${ }^{1,2}$ Y EVA ANDRÉS $^{1}$ \\ ${ }^{1}$ Universidad de Zaragoza \\ ${ }^{2}$ Hospital Clínico Universitario Miguel Servet, Zaragoza
}

\begin{abstract}
Resumen: Mediante el presente estudio examinamos la estructura del Cuestionario Breve de Burnout (CBB; Moreno, Bustos, Matallana y Millares, 1997). El CBB permite evaluar el síndrome de burnout como un proceso cuyos antecedentes serían las características de la tarea y de la organización en la que se trabaja. El burnout se evalúa según las dimensiones clásicas, esto es, cansancio emocional, despersonalización y falta de realización personal. El CBB también evalúa consecuencias del síndrome mediante una escala sobre consecuentes físicos, sociales y psicológicos. Los resultados del análisis de componentes principales, basados en una muestra de docentes, confirmaron la estructura original de las escalas de antecedentes y consecuentes, pero no la del burnout. Según nuestros resultados, la escala de antecedentes permitiría la estimación independiente de características de la organización y de la tarea, pero las escalas burnout y de los consecuentes dependerían de una interpretación general. Fueron encontradas diferencias en función del sexo y nivel de docencia.
\end{abstract}

Palabras clave: Cuestionario Breve de Burnout, CBB, validez factorial, burnout, docentes.

\section{Factorial validity of the Cuestionario Breve de Burnout (Burnout Short Questionnaire) in a sample of teachers in Aragón}

\begin{abstract}
This study examines the structure of the Cuestionario Breve de Burnout (CBB; Moreno, Bustos, Matallana y Millares, 1997) [Burnout Short Questionnaire]. This questionnaire evaluates the burnout syndrome as a process with antecedents being the features of the task and the way the job is organized. Burnout is evaluated from the three classical dimensions: emotional exhaustion, depersonalization, and lack of personal accomplishment. The CBB also assesses the consequences of the syndrome with a scale that measures physical, social, and psychological consequences. The results of the analysis of the principal components in a sample of teachers, confirms the original structure of the antecedents and consequences scales, but not of the burnout scale. According to our study, the antecedents scale allows an independent estimation of the features of the organization and of the task, but the burnout and consequents scales only allow a general interpretation. We encountered differences depending on gender and teaching level.
\end{abstract}

Keywords: Burnout Short Questionnaire, CBB, factorial validity, burnout, questionnaire, teachers.

\section{INTRODUCCIÓN}

Basándonos en los datos proporcionados por la VI Encuesta Nacional de Condiciones de Trabajo (2007), el 22,5\% de los trabajadores consideran que el trabajo afecta significativa-

Recibido: 25-mayo-2008; aceptado: 30-julio-2008.

Correspondencia: Jesús Montero Marín, Plaza Lizana 2-3, $3^{\circ}$ C. C.P., 22002 Huesca.

Correo-e: jmontero@unizar.es

Agradecimientos: Agradecemos al Dr. Bonifacio Sandín y al Dr. Miguel A. Santed (UNED), así como al Dr. Bernardo Moreno (UAM), la revisión del presente trabajo. mente el estado de salud. Entre los problemas que en opinión de este grupo de trabajadores pueden ser atribuibles al trabajo se encuentran: dolores de espalda $(57,6 \%)$, dolores de cuello $(28,1 \%)$, estrés $(27,9 \%)$, dolores de cabeza $(8,5 \%)$, cansancio crónico $(7,2 \%)$, alteraciones del sueño $(6,2 \%)$, depresión $(5,4 \%)$, dificultades respiratorias $(4,9 \%)$, alergias y problemas de la piel $(4,6 \%)$, alteraciones gastrointestinales $(3,4 \%)$, tensión arterial alta $(2,3 \%)$, etc. Todos estos síntomas han sido relacionados con el síndrome de burnout (Gil-Monte, 2005). También se acepta que puedan ser respuestas emo- 
cionales y fisiológicas de estrés que influyen en el estatus de salud (Sandín, 2009).

Los resultados proporcionados por la Encuesta de Calidad de Vida en el Trabajo (2006) apuntan que los profesionales de la educación destacan por su elevado número de quejas en relación al estrés, dolores de espalda, insomnio y depresión. Si atendemos a la sintomatología psicosomática, los resultados de esta última encuesta apuntan que «tardar en dormirse» $(14,4 \%)$, «sensación continua de cansancio» $(12,1 \%)$, «dolores de cabeza» $(11,9 \%), y$ «tensión e irritabilidad» $(10,4 \%)$ alcanzan proporciones considerables entre los docentes. En España, algunos estudios han llegado a obtener altas puntuaciones para el síndrome de burnout en el $40 \%$ de los profesores participantes (Cordeiro, 2001; Manassero, Fornés, Fernández, Vázquez y Ferrer, 1995).

Burnout es un término anglosajón cuya traducción más próxima y coloquial viene a ser «estar quemado», desgastado, exhausto y sin ilusión por el trabajo (Gil-Monte, 2005). Será Freudenberger (1974) quien dará comienzo al desarrollo del constructo burnout como un tipo de estrés generalmente vinculado a profesiones asistenciales o de servicios. Con posterioridad, Maslach y Jackson (1981), basándose en el estudio de los procesos de categorización emocional, definirán el burnout como un síndrome de agotamiento emocional, despersonalización y falta de realización personal en el trabajo. Por agotamiento emocional se entiende una pérdida progresiva de la capacidad de entrega en el trabajo; la despersonalización se compone de actitudes negativas y cínicas hacia el usuario así como hacia la organización donde el profesional ejerce; y la falta de realización personal hace referencia a una persona profesionalmente descontenta, desmotivada e insatisfecha (Seisdedos, 1997). Las primeras manifestaciones clínicas del síndrome vendrían dadas por el cansancio emocional, dando paso posteriormente a la despersonalización y a la baja realización personal (Maslach y Leiter, 1999). En general, se acepta que el síndrome de burnout es el resultado de un proceso de estrés laboral crónico (Gil-Monte, 2005).

En estos últimos años han existido otras diferentes conceptualizaciones del burnout, como el modelo de Farber, cuya validez ha sido estudiada por nuestro grupo recientemente (Montero, García-Campayo y Andres 2008). Otros autores, como Moreno, Bustos, Matallana y Millares (1997), desde una perspectiva psicosocial y recogiendo de manera global la propuesta de Schwab, Jackson y Schuler (1986), han planteado un modelo del síndrome de burnout operacionalizado a través del Cuestionario Breve de Burnout (CBB), basándose en las tres dimensiones clásicas anteriormente citadas, pero incorporando además medidas de factores antecedentes y consecuentes, por lo que consideran el desarrollo del síndrome como un proceso. La dimensión de burnout correspondiente al cansancio emocional en el CBB se entiende como el hecho de estar harto del trabajo, malhumorado cuando se está en él, y por sentirse en general quemado (entendido en términos coloquiales). La falta de realización personal se correspondería en este cuestionario con la ausencia de retos personales en el trabajo y de interés en cuanto al desarrollo profesional, así como por el hecho de hacer un trabajo muy distinto del que se hubiera querido realizar. La despersonalización es contemplada como la percepción de exigencias excesivas y comportamientos irritantes por parte de los usuarios, el distanciamiento o actitud despersonalizada en las relaciones con dichos usuarios, y la sensación de que éstos no reconocen los esfuerzos que se hacen por ellos.

Como factores antecedentes el CBB considera la insatisfacción con la tarea y con la organización. El primero de ellos ha sido confirmado desde el modelo de Janssen, de Jonge y Bakker (1999), al prestar atención a la variedad, autonomía y feedback de la tarea a realizar. El interés por las bases organizacionales del síndrome hunde sus raíces en el modelo de Cherniss (1980), y en la propuesta de Janssen, de Jonge y Bakker (1999) ha sido enfocado hacia las relaciones sociales laborales (apoyo social y participación), así como las condiciones del empleo (salario, seguridad laboral y oportunidades de promoción). En el CBB los aspectos organizacionales se atienden mediante la valoración de las relaciones con el supervisor, con los compañeros, las relaciones personales en el trabajo, el ambiente, y el clima laboral general. 
Respecto a la insatisfacción con la tarea, el CBB tiene en cuenta la identificación personal con el trabajo, el interés que suscita, la responsabilidad y alcance como expectativas de control sobre los resultados en el trabajo (Hernández, Fernández, Ramos y Contador, 2006), así como el aburrimiento y la monotonía experimentados.

Además de los factores antecedentes y de las dimensiones del síndrome en sí mismo, el modelo propuesto por Moreno et al. (1997) a través del CBB, ha subrayado ciertos patrones de relaciones causales del estrés laboral con sus consecuencias. En general, se acepta que las condiciones de trabajo pueden producir consecuencias adversas en la salud y en el bienestar de los empleados (Cooper, 1998; Schaufeli y Enzmann, 1998; Boada, de Diego, Agulló, 2004). En esta línea, el CBB atiende brevemente a consecuencias físicas (psicosomáticas), sociales (relaciones personales y familiares) y psicológicas (rendimiento en el trabajo). De este modo, la operacionalización del CBB podría permitir una evaluación global breve, y por tanto muy operativa, de los elementos presentes en el proceso psicosocial de desarrollo del síndrome de burnout o desgaste profesional. De todos modos, el autor de la escala señala que el $\mathrm{CBB}$ ha sido diseñado para valorar las escalas generales de antecedentes, burnout y consecuentes, y no las subescalas que lo integran por separado, siendo que éstas únicamente nos darían el soporte teórico necesario para comprender la estructura del cuestionario.

Dada la prevalencia del burnout, de su sintomatología asociada entre la población docente española, y por extensión, la necesidad de contar con instrumentos adecuados para evaluar dicho síndrome entre el profesorado, el objetivo principal que ha guiado el presente estudio ha sido explorar la validez factorial de la estructura teórica del CBB, así como la consistencia interna de sus escalas, en una muestra de profesores aragoneses de enseñanza infantil, primaria y secundaria. Como objetivo secundario nos propusimos valorar las posibles diferencias en función del sexo y del nivel de docencia.

\section{MÉTODO}

Utilizamos el método correlacional con un diseño de corte transversal. Las medidas se llevaron a cabo a través de la técnica del autoinforme, previo consentimiento informado de los participantes elegidos incidentalmente.

\section{Participantes}

La muestra utilizada estuvo compuesta por 309 docentes españoles activos en el año 2006, pertenecientes a 17 colegios públicos aragoneses con niveles de enseñanza infantil, primaria y secundaria. La selección de los colegios aragoneses fue incidental y la muestra se obtuvo gracias a la colaboración de aquellos profesores que accedieron voluntariamente a participar en el estudio. Aproximadamente el $25 \%$ de los colegios pertenecían a zonas urbanas, mientras que el $75 \%$ al ámbito rural. En cuanto al sexo de los participantes, el $75 \%$ fueron mujeres y el $25 \%$ hombres. La media de edad fue 37,3 años $(D T=4,8 ;$ rango $=25-64)$. El porcentaje de participantes según el nivel de docencia fue del $17 \%$ para los dedicados a educación infantil, del $10 \%$ para aquellos dedicados a infantil y primaria de forma simultánea, del 39\% para los dedicados a educación primaria y del 34\% para los de secundaria. En cuanto al tipo de contrato, el $24 \%$ fueron trabajadores interinos y el $76 \%$ trabajadores con contrato fijo.

\section{Instrumentos}

El protocolo administrado incluía un cuestionario de elaboración propia sobre las características sociodemográficas y laborales, y el cuestionario CBB. Éste se compone de 21 items, cuyas respuestas son estimadas a partir de una escala tipo Likert, con cinco opciones de respuesta, valoradas de 1 a 5 en este orden: «nada», «muy poco», «algo», «bastante», «mucho» (items 13, 15, 16, 17, 19); «en ninguna ocasión», «raramente», «algunas veces», «frecuentemente», «en la mayoría de ocasiones» (items 1, 2, 3, 4, 7, 8, 14, 20, 21); «totalmente en desacuerdo», «en desacuerdo», «indeciso», 
«de acuerdo», «totalmente de acuerdo» (items $5,6,10,11,12)$; «nunca», «raramente», «algunas veces», «frecuentemente», «siempre» (items 9, 18). El CBB incluye las siguientes escalas: sindrome de burnout (incorpora las 3 dimensiones clásicas: cansancio emocional, realización personal y despersonalización), antecedentes (incorpora las dimensiones de organización e insatisfacción con la tarea), y consecuentes.

En el trabajo de Moreno et al. (1997) los valores de consistencia interna del CBB fueron como sigue: síndrome de burnout: $\alpha=0,74$ (9 items); cansancio emocional: $\alpha=0,82$ (3 items); realización personal: $\alpha=0,64$ (3 items); despersonalización: $\alpha=0,35$; antecedentes: $\alpha=0,77$ (9 items); organización: $\alpha=0,78$ (4 items); insatisfacción con la tarea: $\alpha=0,69$ (5 items); y consecuentes: $\alpha=0,54$ (3 items). (Para la descripción de los items véanse las tablas 1, 2 y 3 ).

Según Moreno et al. (1997), la validez convergente de la escala global de burnout del CBB respecto al criterio establecido mediante el MBI (tomados ambos de manera unidimensional) adquiere un valor de $r=0,688$, significativa a un nivel $p=0,001$. Igualmente, las escalas de antecedentes y consecuentes del CBB muestran niveles significativos de asociación con el síndrome de burnout en general, medido tanto por el CBB, como a través del MBI. Así, la relación entre antecedentes y burnout en el CBB es de

Tabla 1: Matriz de factores rotados de la escala antecedentes (CBB). Componentes principales, rotación varimax

\begin{tabular}{lcc}
\hline \multirow{2}{*}{ Items } & \multicolumn{2}{c}{ Factores } \\
\cline { 2 - 3 } & $(1)$ & $(2)$ \\
\hline 4. Mi supervisor me apoya en las decisiones que tomo & 0,46 & 0,16 \\
8. Los compañeros nos apoyamos en el trabajo & $\mathbf{0 , 8 3}$ & 0,06 \\
9. Las relaciones personales que establezco en el trabajo son gratificantes & $\mathbf{0 , 7 8}$ & 0,12 \\
16. Me gusta el ambiente y el clima de mi trabajo & $\mathbf{0 , 8 0}$ & 0,19 \\
2. Me siento identificado con mi trabajo & 0,44 & $\mathbf{0 , 5 7}$ \\
6. Mi trabajo actual carece de interés & 0,29 & $\mathbf{0 , 7 6}$ \\
10. Dada la responsabilidad que tengo en mi trabajo no conozco bien los resultados & 0,11 & $\mathbf{0 , 5 7}$ \\
14. Mi trabajo es repetitivo & $-0,06$ & $\mathbf{0 , 7 2}$ \\
20. Mi trabajo me resulta muy aburrido & 0,23 & $\mathbf{0 , 7 5}$ \\
\hline
\end{tabular}

Nota . Factor 1 = Organización; Factor 2 = Tarea. En negrita los pesos superiores a 0,50.

Tabla 2: Matriz de factores rotados de la escala de burnout (CBB) con una solución de dos factores. Componentes principales, rotación varimax

\begin{tabular}{lcc}
\hline Items & \multicolumn{2}{c}{ Factores } \\
\cline { 2 - 3 } 1. En general estoy más bien harto de mi trabajo & $(1)$ & $(2)$ \\
7. Cuando estoy en mi trabajo me siento de mal humor & $\mathbf{0 , 8 0}$ & 0,17 \\
15. Estoy quemado por mi trabajo & $\mathbf{0 , 8 0}$ & 0,00 \\
5. Mi trabajo profesional me ofrece actualmente escasos retos personales & $\mathbf{0 , 7 4}$ & 0,36 \\
12. Mi interés por el desarrollo profesional es actualmente muy escaso & 0,23 & $\mathbf{0 , 7 7}$ \\
19. El trabajo que hago dista de ser el que yo hubiera querido & 0,36 & $\mathbf{0 , 6 2}$ \\
3. Los usuarios de mi trabajo tienen frecuentemente exigencias excesivas & $\mathbf{0 , 5 2}$ & 0,47 \\
11. Las personas a las que tengo que atender reconocen muy poco los esfuerzos & $\mathbf{0 , 6 1}$ & 0,08 \\
18. Procuro despersonalizar las relaciones con los usuarios de mi trabajo & 0,37 & 0,39 \\
\hline
\end{tabular}

Nota. Se han agrupado los items según la propuesta teórica original. Aparecen en negrita los pesos superiores a 0,50. 
Tabla 3: Matriz de factores rotados para la escala de burnout (CBB) con una solución de tres factores. Componentes principales, rotación varimax

\begin{tabular}{lrrr}
\hline & & \multicolumn{2}{c}{ Factores } \\
\cline { 2 - 4 } Items & (1) & (2) & (3) \\
& C. Emoc. & F. Realiz. Despers. \\
\hline 1. En general estoy más bien harto de mi trabajo & $\mathbf{0 , 7 4}$ & 0,35 & $-0,01$ \\
7. Cuando estoy en mi trabajo me siento de mal humor & $\mathbf{0 , 7 8}$ & 0,19 & $-0,08$ \\
15. Estoy quemado por mi trabajo & $\mathbf{0 , 6 1}$ & $\mathbf{0 , 5 7}$ & $-0,00$ \\
5. Mi trabajo profesional me ofrece actualmente escasos retos personales & 0,07 & $\mathbf{0 , 7 6}$ & 0,31 \\
12. Mi interés por el desarrollo profesional es actualmente muy escaso & 0,14 & $\mathbf{0 , 8 2}$ & 0,02 \\
19. El trabajo que hago dista de ser el que yo hubiera querido & 0,39 & $\mathbf{0 , 5 9}$ & 0,11 \\
3. Los usuarios de mi trabajo tienen frecuentemente exigencias excesivas & $\mathbf{0 , 7 1}$ & $-0,03$ & 0,30 \\
11. Las personas a las que tengo que atender reconocen muy poco los esfuerzos & 0,39 & 0,23 & 0,41 \\
18. Procuro despersonalizar las relaciones con los usuarios de mi trabajo & $-0,02$ & 0,11 & $\mathbf{0 , 8 6}$ \\
\hline
\end{tabular}

Nota C. Emoc.= Cansancio Emocional. F. Realiz.= Falta de Realización. Despers.= Despersonalización. Se han separado los items según la propuesta teórica original. En negrita se indican los pesos superiores a 0,50.

$r=0,60(p<0,001), \mathrm{y}$ con el MBI de $r=0,54$ $(p<0,001)$. La relación entre consecuentes $\mathrm{y}$ burnout en el CBB es de $r=0,47(p<0,001)$ y con el MBI de $r=0,46(p<0,001)$.

\section{Análisis de datos}

Para examinar la estructura del CBB se llevaron a cabo análisis factoriales (método de componentes principales). Para la extracción del número de factores utilizamos el criterio de Kaiser (1960) y el test de scree de Cattell (1966). Aplicamos rotación ortogonal (método varimax) para obtener la máxima independencia de los factores. Para confirmar la viabilidad del análisis factorial exploratorio (AFE) nos aseguramos de que las correlaciones en cada matriz presentaban un alto porcentaje de puntuaciones $>0,30$ y que todas las variables tenían correlaciones significativas; que el determinante era muy bajo pero no nulo; que el índice KMO presentaba un valor mayor de 0,70; que los valores absolutos de los coeficientes de las matrices anti-imagen eran bajos y los de los coeficientes de adecuación muestral elevados, por encima de 0,80 , y que el resultado de la prueba de esfericidad de Barlett daba un resultado significativo. El análisis de fiabilidad de las escalas se llevó a cabo mediante el $\alpha$ de Crombach, $\mathrm{y}$ la relación de cada item respecto a su escala de pertenencia se cuantificó a través del coeficiente de discriminación o correlación item-resto. La relación entre las escalas se evaluó a través del coeficiente de correlación de Pearson, y las diferencias entre los subgrupos fueron cuantificadas mediante la prueba $t$ de comparación de medias.

\section{Procedimiento}

Un psicólogo clínico se encargó de explicar los objetivos de la investigación a 25 equipos directivos de varios colegios de Aragón (España). Se repartieron 400 protocolos entre los profesores de los 17 colegios que mostraron su consentimiento y se asignó a cada sujeto participante una clave numérica, para su corrección salvando el anonimato. Aquellos participantes que desearon conocer sus puntuaciones pudieron hacerlo de manera anónima a través de dicha clave numérica. Los jefes de estudio de cada centro se encargaron de la recogida de los cuestionarios mediante una caja de cartón cerrada con una única apertura posteriormente precintada, por donde se introducían las encuestas cumplimentadas. Todos los participantes cumplimentaron el protocolo durante la segunda quincena de mayo de 2006. El análisis de los datos fue llevado a cabo mediante el paquete estadístico SPSS.13. 


\section{RESULTADOS}

\section{Análisis Factorial del CBB}

El resultado del análisis factorial de los items que forman la escala general de antecedentes arrojó una solución formada por dos factores, los cuales explicaron conjuntamente el $55,1 \%$ de la varianza total. El primer factor, con un autovalor de 3,55 explicaba el 39,53\% de la varianza total inicial; y el segundo, con un autovalor de 1,40 el $15,5 \%$. Ambos autovalores cumplían el criterio de Kaiser (1960). Los autovalores del resto de los componentes eran menores a la unidad. El test de scree sugería igualmente que la estructura correspondía a dos factores.

Nuestros resultados muestran una solución factorial similar a la obtenida por Moreno et al. (1997). Es de señalar que todos los items presentaron un peso factorial superior a 0,30 (Comrey, 1985) en su factor de pertenencia. El primer factor (organización) estuvo constituido por los items 4, 8, 9 y 16; y el segundo factor (tarea) por los items 2, 6, 10, 14 y 20 (véase la Tabla 1).

El análisis factorial de la escala del síndrome de burnout sugería una solución de dos factores (sólo obtuvimos 2 factores con autovalores $\geq 1$ ), los cuales explicaban el 53,63\% de la varianza total, con unos autovalores de 3,68 en el primer factor $(40,92 \%$ de la varianza inicial total) y 1,14 en el segundo (23,70\%). El gráfico de sedimentación manifestó un claro punto de inflexión en la solución de dos factores. No obstante, dado que la estructura teórica del constructo era de tres factores, complementariamente examinamos la solución de 3 factores (el tercer valor tenía un autovalor de sólo 0,89). Dicho tercer factor explicaba el 9,95\% de la varianza inicial total (los tres factores explicaban el $63,58 \%$ de la varianza total). Los datos correspondientes a estos análisis son presentados en la Tabla 2 (solución de dos factores) y en la Tabla 3 (solución de 3 factores). Puede apreciarse que tanto la estructura de dos factores como la de tres factores son poco consistentes ya que existen items que saturan de forma elevada en factores irrelevantes (factores no vinculados teóricamente a dichos items). Aunque los datos que presentamos en la Tabla 3 replican en cierto grado los resultados referidos por Moreno et al. (1997), debe tenerse en cuenta la limitación señalada arriba sobre la validez de los tres factores, así como también algunas inconsistencias en la configuración factorial (véanse las Tablas 2 y 3 ).

El análisis factorial de los items de la escala de consecuentes arrojó una solución de un solo factor que explicaba el $62,56 \%$ de la varianza. Dicho factor presentó un autovalor de 1,87, siendo los pesos factoriales de los tres items superiores a 0,75 .

\section{Análisis de fiabilidad y correlaciones entre las escalas}

En la Figura 1 presentamos nuestros resultados sobre la consistencia interna de las escalas generales del CBB. Los valores obtenidos al eliminar cada item por separado en la escala de antecedentes fueron menores en todos los casos excepto para el item $\mathrm{n}^{\circ} 4$ ( $« \mathrm{Mi}$ supervisor me apoya en las decisiones que tomo»), cuya eliminación hacía subir la fiabilidad hasta $\alpha=$ 0,79 . Para esta escala los coeficientes de discriminación fueron superiores a 0,50 en todos los casos excepto para los items $4(0,33), 10$ «Dada la responsabilidad que tengo en mi trabajo, no conozco bien los resultados y el alcance del mismo» $(0,35)$, y 14 «Mi trabajo es repetitivo» $(0,32)$. Por otra parte, los valores de consistencia interna obtenidos para la subescala organización fue de $\alpha=0,70$ y para la de insatisfacción con la tarea de $\alpha=0,74$.

La consistencia interna obtenida al eliminar cada item por separado de la escala global de burnout, fue menor en todos los casos excepto

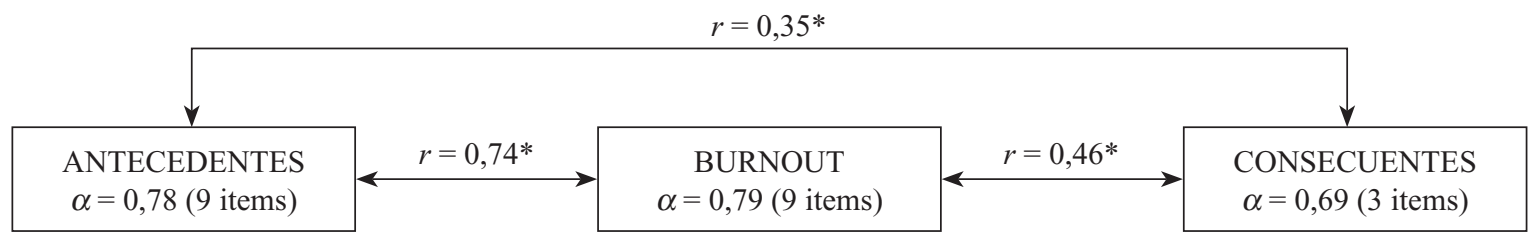

Figura 1. Modelo basado en el Cuestionario Breve de Burnout $(* p<0,01)$. 
Tabla 4: Medias y desviaciones típicas de las escalas del CBB para la muestra total y en función del sexo y del nivel de docencia (infantil-primaria vs. secundaria)

\begin{tabular}{|c|c|c|c|c|}
\hline \multirow{2}{*}{$C B B$} & Total $(N=309)$ & Varones $(n=75)$ & Mujeres $(n=230)$ & \multirow{2}{*}{$t$} \\
\hline & Media $(D T)$ & Media $(D T)$ & Media $(D T)$ & \\
\hline ANTECEDENTES & $18,69(4,55)$ & $20,31(4,72)$ & $18,17(4,35)$ & $3,48 * *$ \\
\hline Organización & $8,44(2,56)$ & $9,00(2,73)$ & $8,27(2,45)$ & $2,08^{*}$ \\
\hline Tarea & $10,26(2,81)$ & $11,37(2,63)$ & $9,88(2,79)$ & $4,03 * * *$ \\
\hline BURNOUT & $21,83(5,14)$ & $23,29(4,88)$ & $21,38(5,15)$ & $2,75^{* *}$ \\
\hline \multirow[t]{3}{*}{ CONSECUENTES } & $7,43(2,26)$ & $7,16(2,11)$ & $7,55(2,28)$ & $-1,31$ \\
\hline & & $\operatorname{Infan}-\operatorname{Prim}(n=203)$ & Secundaria $(n=98)$ & \multirow{2}{*}{$t$} \\
\hline & & Media $(D T)$ & Media $(D T)$ & \\
\hline ANTECEDENTES & & $17,87(4,02)$ & $20,433(5,10)$ & $-4,18 * * *$ \\
\hline Organización & & $8,27(2,49)$ & $8,793(2,62)$ & $-1,62$ \\
\hline Tarea & & $9,58(2,36)$ & $11,673(3,22)$ & $-5,63 * * *$ \\
\hline BURNOUT & & $20,71(4,47)$ & $24,191(5,70)$ & $-5,17 * * *$ \\
\hline CONSECUENTES & & $7,39(2,04)$ & $7,551(2,67)$ & $-0,51$ \\
\hline
\end{tabular}

Nota. Infan-Prim. $=$ Infantil y Primaria. ${ }^{*} p<0,05 ; * * p<0,01 ; * * *<<0,001$.

para el item 18 («Procuro despersonalizar las relaciones con los usuarios de mi trabajo»), cuya eliminación la hacía subir hasta $\alpha=0,81$. Los coeficientes de discriminación para los items que componen dicha escala general de burnout fueron superiores a 0,40 en todos los casos, excepto para el item $18(0,20)$. No se calculó la fiabilidad de las subdimensiones propuestas por Moreno et al. (1997) para la escala de burnout, puesto que dicha estructura no ha sido apoyada empíricamente por nuestro estudio.

Los valores para la escala de consecuentes al eliminar cada item por separado fueron inferiores al valor presentado para la escala total. Los coeficientes de discriminación para los tres items que componen esta escala fueron superiores a 0,45 en todos los casos.

Los resultados de la relación entre las escalas generales mediante el coeficiente de correlación de Pearson, arrojaron valores significativos en todos los casos, tal y como se presentan en la Figura 1.

\section{Datos normativos y diferencias entre grupos de género y nivel de educación}

En la Tabla 4 se presentan las medias y desviaciones típicas para las escalas y subescalas del CBB replicadas en nuestro estudio. Al dividir la media de las puntuaciones directas, entre el $n^{\circ}$ de items que conforman cada una de ellas, obtuvimos la media escalar, que en el caso de los antecedentes fue de 2,07 $(D T=0,50)$; de $2,11(D T=0,64)$ para la organización y de 2,05 $(D T=0,56)$ para la tarea; de $2,42(D T=0,57)$ para la escala de burnout; y de $2,47(D T=0,75)$ para los consecuentes. Dichas puntuaciones medias escalares tuvieron valores inferiores a 3 , que viene a ser el punto central de la escala de respuesta. En la Tabla 4 se presentan también los valores de la prueba $t$ asociados a las diferencias en las puntuaciones de las escalas del $\mathrm{CBB}$, en función del sexo y del nivel de docencia.

\section{DISCUSIÓN}

El análisis factorial de los 9 items de la escala correspondiente a los antecedentes del CBB replicó la propuesta de Moreno et al. (1997), puesto que en nuestro estudio dicha escala se reestructuró en otras dos, denominadas organización e insatisfacción con la tarea. La primera de ellas, con una adecuada consistencia interna, gira en torno a cuestiones como el clima laboral, las relaciones personales en el 
trabajo y el apoyo por parte de los compañeros y el supervisor. La segunda dimensión que conforma la escala trata consistentemente aspectos como el desinterés por el trabajo, la monotonía de las tareas, la no identificación con el trabajo que se realiza y la falta de control sobre los resultados. La fiabilidad de la escala general de antecedentes en nuestro estudio fue buena, aunque el hecho de que el item 4 («mi supervisor me apoya en las decisiones que tomo») haga disminuir su valor, sugiere la posibilidad de que dicho item pueda resultar reactivo.

Para replicar la estructura teórica de la escala de burnout fue necesario forzar la solución del AFE a tres factores, el tercero, aunque se acercó, no llegó a cumplir la regla de Kaiser (1960). Por tanto, no parece que nuestros datos respalden una estructura tridimensional en dicha escala, sino que por el contrario apuntan hacia una solución más sencilla de dos factores. El primero de ellos estaría constituido por todos los items propuestos inicialmente para la dimensión cansancio emocional (items 1, 7, 15), los cuales versan en torno al hecho de estar harto, quemado y de mal humor en el trabajo, más un item propuesto para despersonalización $\left(n^{\circ} 3\right)$, que trata sobre las frecuentes y excesivas exigencias de los usuarios. Por otra parte, el segundo de los factores lo formarían dos items propuestos inicialmente para falta de realización personal (items 5 y 12), que hacen referencia a los escasos retos personales que ofrece el trabajo y al poco interés del sujeto por su desarrollo profesional, así como un item perteneciente a despersonalización (item 18), en el que se pone de manifiesto el interés por despersonalizar las relaciones con los usuarios. Parece claro que el sentido del primer factor tiene que ver con la irritación y el enojo que siente el sujeto en su puesto de trabajo, mientras que el segundo factor tendría que ver con la falta de desarrollo a nivel personal, social y profesional. En el estudio de Moreno et al. (1997) la dimensión despersonalización presentó problemas de consistencia interna y reparto de uno de los items, pero en nuestro estudio dicha dimensión termina por diluirse en los otros dos factores. Este resultado está en la línea de los problemas planteados por el MBI (Gil-Monte, 2005), siendo que algunos autores han llegado a señalar que el núcleo del burnout podría encontrarse en las otras dos dimensiones (Topa y Morales, 2005), perspectiva que puede conducir a desconsiderar dicha dimensión, o a redefinirla, tal y como se propone en el MBI-GS (Maslach, Jackson y Leiter, 1996).

Los resultados del análisis de consistencia de la escala general de burnout, eliminando los items uno a uno, parecen apoyar la idea de unidimensionalidad (excepto para el item 18: «procuro despersonalizar las relaciones con los usuarios de mi trabajo»). Este resultado estaría de acuerdo con la utilización de una puntuación global de burnout como suma de sus componentes, defendida por autores como Burke, Deszca y Schearer (1984) o Fimien (1984). Dichos resultados nos animan a pensar que, por el momento, lo más prudente es obtener una única puntuación para la escala general de burnout en el CBB, en el mismo sentido que lo enunciado por su propio autor. No obstante, coincidimos con éste en que será el ámbito de aplicación quien dictará la pertinencia de utilizar o no dicha simplificación. Tal vez una valoración de ésta índole resultará más apropiada en ambientes organizacionales (Olabarría y Mansilla, 2007) que en clínicos, en los que bajo nuestro punto de vista resulta importante la identificación de perfiles.

Los resultados de los análisis sobre la escala consecuentes señalan que se trata de una escala consistente, aunque tal vez demasiado breve. El espectro de consecuencias o síntomas al que alude el síndrome de burnout es tan amplio, que resulta difícil pensar que tres items puedan recoger adecuadamente todas sus manifestaciones. No obstante, resulta interesante el esquema de tres tipos de síntomas (físicos, sociales y psicológicos).

Por otra parte, las relaciones significativas entre las escalas generales del CBB permiten mantener el marco teórico del burnout como un proceso, aunque pensamos que sería bueno contrastar la naturaleza de estas relaciones mediante estudios de carácter longitudinal, tratando de aclarar si el tipo de relaciones que presentan las escalas es de orden causal.

Tras comparar las puntuaciones medias entre los grupos de varones y mujeres, hemos constatado que los varones obtenían valores 
significativamente más elevados en la escala de antecedentes (y en las subescalas organización y tarea), así como en la escala de burnout, pero no en la de consecuentes. Por otra parte, también fueron encontradas diferencias significativas en función del nivel de docencia en la escalas antecedentes (y también en la subescala relativa a la insatisfacción con la tarea), así como en la escala de burnout, siendo que en todos los casos los profesores de enseñanza secundaria presentaron puntuaciones más elevadas. El efecto de la organización resultó ser similar tanto para el caso de infantil y primaria, como para el de secundaria. En este caso tampoco fueron encontradas diferencias en los consecuentes del burnout. No sabríamos decir si ambos resultados se deben a una ausencia real de diferencias en el nivel de consecuencias del síndrome, o bien a que dicha escala no recoge adecuadamente todas las manifestaciones de dichas consecuencias.

Con todo, el CBB parece un cuestionario adecuado para valorar brevemente el proceso de burnout en las escalas generales de antecedentes, burnout y consecuentes. A la luz de nuestros resultados podría incluso valorar las subescalas organización y tarea de manera independiente.

\section{REFERENCIAS}

Boada, J., de Diego, R., y Agulló, E. (2004). El burnout y las manifestaciones psicosomáticas como consecuentes del clima organizacional y de la motivación laboral. Psicothema, 16, 125-131.

Burke, R.J., Deszca, E., \& Schearer, J. (1984). Career orientations and burnout in police officers. Canadian Journal of Administrative Science, 1, 179-194.

Cattell, R. B. (1966). The scree test for the number of factors. Multivariate Behavioral Research, 1, 245-76.

Cherniss, C. (1980). Staff burnout: Job stress in the human services. Beverly Hills: Sage.

Comrey, A.L. (1985). Manual de Análisis Factorial. Madrid. Cátedra.

Cooper, C. (1998). Introduction. En C.L. Cooper (Ed.), Theories of organizational stress (pp. 1-5). Oxford: Oxford University Press.

Cordeiro, J. A. (2001). Prevalencia del Síndrome de Burnout en los Profesores de Primaria de la Zona Educativa de la Bahía de Cádiz. Tesis doctoral no publicada.
Fimien, M. J. (1984). The development of an instrument to measure occupational stress in teachers: The Theacher Stress Inventory. Journal of Occupational Psychology, 57, 277-293.

Freudenberger, H. (1974). Saff burn-out. Journal of Social Issues, 30, 159-166.

Gil-Monte, P. R. (2005). El síndrome de quemarse por el trabajo (burnout). Una enfermedad laboral en la sociedad del bienestar. Madrid: Pirámide.

Hernández, L., Fernández, F., y Contador, I. (2006). Burnout, expectativas de control y afrontamiento en un grupo de funcionarios de prisiones. Revista de Psicopatología y Psicología Clínica, 11, 155-164.

Janssen, P., de Jonge, J., \& Bakker, A. (1999). Especific determinats of intrinsic work motivation, burnout and turnover intentions: A study among nurses. Journal of Advanced Nursing, 29, 1360-1369.

Kaiser, H. F. (1960). The application of electronic computers to factor analysis. Educational and Psychological Measurement, 20, 141-151.

Manassero, M. A., Fornés, J., Fernández, M. C., Vázquez, A., y Ferrer, V. A. (1995). «Burnout» en la enseñanza. Análisis de su incidencia y factores determinantes. Revista de Educación, 308, 241-266.

Maslach, C., \& Jackson, S.E. (1981). Maslach Burnout Inventory. Palo Alto, CA: Consulting Psychologists Press.

Maslach, C., Jackson, S. E. \& Leiter, M. P. (1996). Maslach Burnout Inventory. Palo Alto, CA: Consulting Psychologist Press.

Maslach, C. \& Leiter, M.P. (1999). Teacher burnout: A research agenda. En R.Vandenberghe y A.M. Huberman (Eds.), Understanding and preventing teacher burnout (pp. 295-303). New York: Cambridge University Press.

Ministerio de Trabajo y Asuntos Sociales (2006). Encuesta de Calidad de Vida en el Trabajo (ECVT). Disponible en http://www.mtas.es/Estadisticas/ecvt/Ecvt2006/ index.htm

Ministerio de Trabajo y Asuntos Sociales (2007). VI Encuesta Nacional de Condiciones de Trabajo. Instituto Nacional de Seguridad e Higiene en el Trabajo. Disponible en http://www.mtas.es/insht/statistics/viencuesta.pdf

Moreno, B., Bustos, R., Matallana, A., y Millares, T. (1997). La evaluación del Burnout. Problemas y alternativas. El CBB como evaluación de los elementos del proceso. Revista de Psicología del Trabajo y de las Organizaciones, 13, 185-207.

Montero, J., García-Campayo, J., y Andrés, E. (2008). Análisis exploratorio de un modelo clínico basado en tres tipos de burnout. Cuadernos de Medicina Psicosomática, 88, 41-49.

Olabarria, B., y Mansilla, F. (2007). Ante el burnout: Cuidados a los equipos de salud mental. Revista de Psicopatología y Psicología Clínica, 12, 1-14. 
Sandín, B. (2009). El estrés. En A. Belloch, B. Sandín, y F. Ramos. Manual de Psicopatología (ed. Rev. vol 2, pp. 3-42). Madrid: McGraw Hill.

Schaufeli, W., \& Enzmann, D. (1998). The burnout companion to study and practice. A critical analysis. London: Francis y Taylor.

Schwab, R.L., Jackson, S.E., \& Schuler, R.S. (1986). Educator burnout: sources and consecuences. Educational Research Quartely, 10, 14-30.
Seisdedos, N. (1997). MBI. Inventario de«Burnout» de Maslach: manual. Madrid: TEA.

Topa, G., y Morales, J. (2005). Determinantes específicos de la satisfacción laboral, el burnout y sus consecuencias para la salud: un estudio exploratorio con funcionarios de prisiones. International Journal of Psychology and Psychological Therapy, 5, 73-83. 